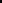




\title{
PROTECTION OF A TEST MAGNET WOUND WITH A Ag/BSCCO HIGH-TEMPERATURE SUPERCONDUCTOR
}

\author{
Lawrence Dresner \\ Fusion Energy Division \\ Oak Ridge National Laboratory
}

October 1992

\author{
Research conducted under \\ Cooperative Agreement No. HTSPC-007 \\ as authorized by the \\ U.S. Department Of Energy \\ under contract DE-AC05-84OR21400 \\ w'ith Martin Marietta Energy Systems, Inc.
}




\section{CONTENTS}

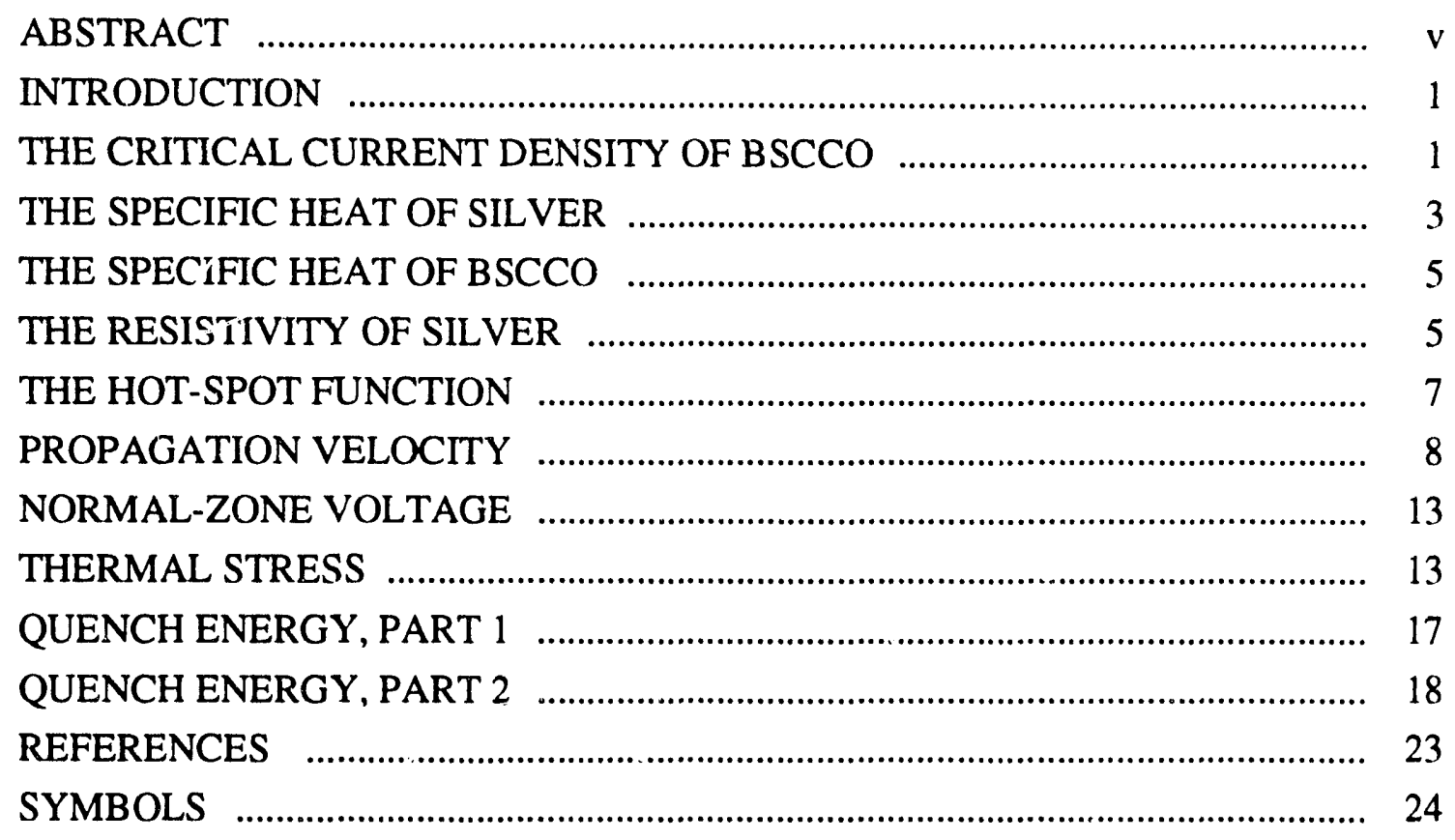




\begin{abstract}
The properties of $\mathrm{Ag} / \mathrm{BSCCO}$ conductor are studied and used to evaluate the feasibility of detecting normal zones in the conductor before the hot-spot temperature reaches critical levels. The results are applied to the protection of a 5-kJ test magnet.
\end{abstract}




\section{INTRODUCTION}

The 5-kJ test magnet being proposed by Superconductivity, Inc., is to have a bore radius of $7.5 \mathrm{in}$. and a width and build each of 3 in. The conductor, to be made by American Superconductor, is to be a multifilamentary Ag/BSCCO wire, operated in the temperature range $20-40 \mathrm{~K}$. The overall current density, chosen to be $2.5 \mathrm{kA} / \mathrm{cm}^{2}$, will produce a central field of $0.4 \mathrm{~T}$ and a maximum field at the winding of $0.9 \mathrm{~T}$.

Oak Ridge National Laboratory has been commissioned to study the protection of this magnet. Owing to (1) the low current densities at which high-temperature superconductors must presently be operated, (2) the substantial difference between their operating and critical temperatures, and (3) their rather large heat capacities between these temperatures, the growth of normal zones is rather slow. This greatly complicates the detection of incipient normal zones. On the other hand, the low current density and high heat capacity cause the hot-spot temperature to rise slowly, so a long time may be available for the detection of normal zones. The purpose of this work is to study the competition between these countervailing influences and decide if it is possible to detect normal zones before the hot-spot temperature reaches harmful levels.

The next few sections of this report are devoted to determining the critical current density of the BSCCO superconductor as a function of field and temperature, the specific heats of silver and BSCCO as functions of temperature, and the electrical resistivity of silver as a function of field and temperature. Then, we calculate the adiabatic hot-spot function as a function of hot-spot temperature and the residual resistance ratio of the silver. From the hot-spot function and the current density, we estimate the time available for detecting a normal zone. With this time and the velocity of growth, we then find the largest voltage that will be available for detecting a normal zone. We conclude that this voltage is large enough to be detected reliably.

A list of symbols is provided as an appendix.

\section{THE CRITICAL CURRENT DENSITY OF BSCCO}

Figure 1 shows the intragrain critical current density $J_{C}$ as a function of the field $B$ and the temperature $T$ for a field parallel to the cryst ! c-axis. $^{1}$ Shown in Fig. 2, plotted against temperature, are values taken from this graph for $B=1 \mathrm{~T}$. The linear fit in the range $20-40 \mathrm{~K}$ extrapolates to zero at $45 \mathrm{~K}$, and we choose this value as the effective critical temperature. We use such a linear approximation to $J_{c}$ as a function of $T$ in order to follow the classical theory of applied superconductivity, which is based on such a linear dependence.

The intragrain critical current density is much larger than the actual current density that can be attained in contemporary $\mathrm{Ag} / \mathrm{BSCCO}$ conductors. Recent data of Aihara et al. ${ }^{2}$ and Miller et al. ${ }^{3}$ imply a $J_{i ;}$ for the BSCCO of roughly $5 \mathrm{kA} / \mathrm{cm}^{2}$ at $20 \mathrm{~K}$ and $1 \mathrm{~T}$. 


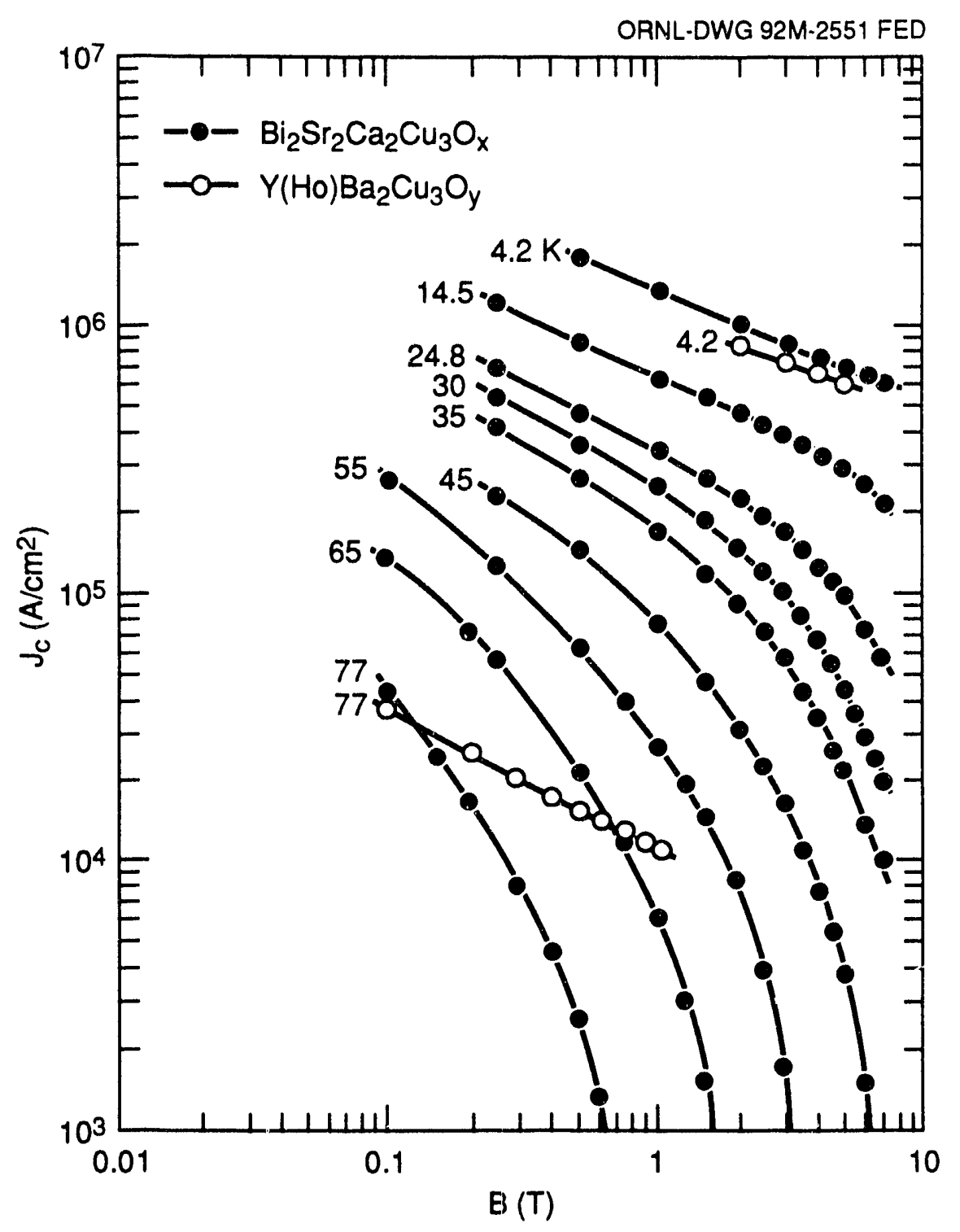

Fig. 1. The intragrain critical current density $J_{\mathrm{c}}$ as a function of field and temperature for a field parallel to the crystal c-axis. From Ref. 1.

Recently Kroeger has reported $10 \mathrm{kA} / \mathrm{cm}^{2}$ at $20 \mathrm{~K}$ and $3 \mathrm{~T}$ (see ref. 4). These values are quite consistent with the assumed overall current density of $2.5 \mathrm{kA} / \mathrm{cm}^{2}$ if we choose an operating temperature of $20 \mathrm{~K}$. Therefore, for the sake of estimation, we choose

$$
\begin{aligned}
& T_{\mathrm{b}}=20 \mathrm{~K}, \quad T_{\mathrm{c}}=45 \mathrm{~K}, \\
& J_{\mathrm{c}}(20 \mathrm{~K}, 1 \mathrm{~T})=5 \mathrm{kA} / \mathrm{cm}^{2} \quad \text { (BSCCO only). }
\end{aligned}
$$




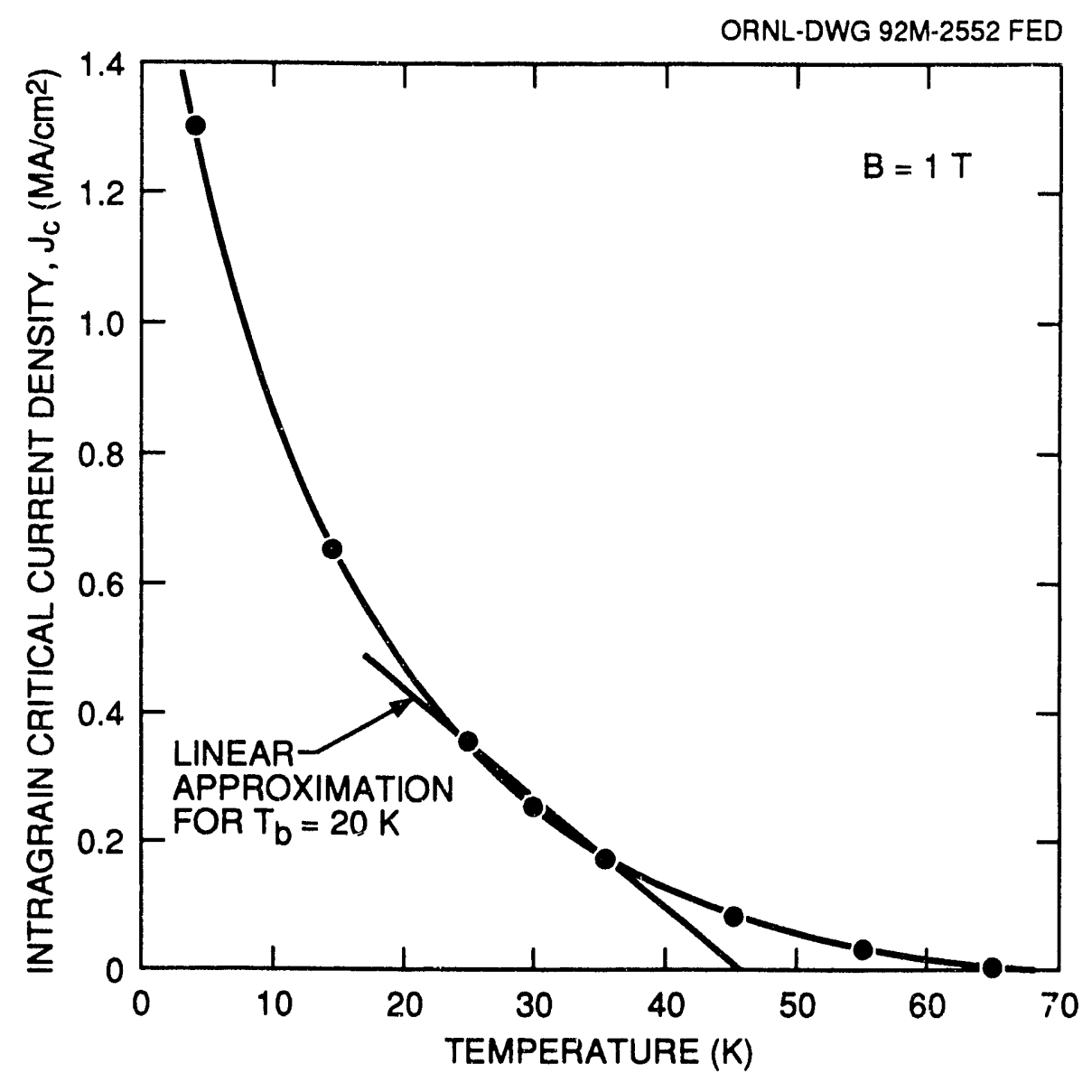

\section{THE SPECIFIC HEAT OF SILVER}

We express the specific heat of silver as the sum of two contributions, the electronic (Sommerfeld) contribution and the phonon (Debye) contribution.

According to Debye's theory of specific heats, the phonon contribution to the specific heat $C_{\mathrm{v}}$ of a monatomic solid is given by

$$
\frac{C_{\mathrm{v}}}{R}=12\left(\frac{3}{z^{3}} \int_{0}^{z} \frac{t^{3}}{e^{t}-1} d t\right)-\frac{9 z}{e^{z}-1}, \quad z=\frac{1}{x}=\frac{\theta}{T},
$$

where $R=8.317 \mathrm{~J} \cdot \mathrm{mol}^{-1} \cdot \mathrm{K}^{-1}$ is the universal gas constant, $T$ is the absolute temperature, and $\theta$ is the Debye temperature. The quantity in the parentheses has been tabulated by Abramowitz and Stegun. ${ }^{5}$ Figure 3 shows a curve of $C_{\mathrm{v}}$ vs $x=T / \theta$ calculated using Eq. (2) and lines representing the two limits

$$
T \ll \theta(\text { Debye }), \quad C_{\mathrm{v} 1}=\frac{108 \pi^{4}}{45} R x^{3}=1944 x^{3} \mathrm{~J} \cdot \mathrm{mol}^{-1} \cdot \mathrm{K}^{-1},
$$




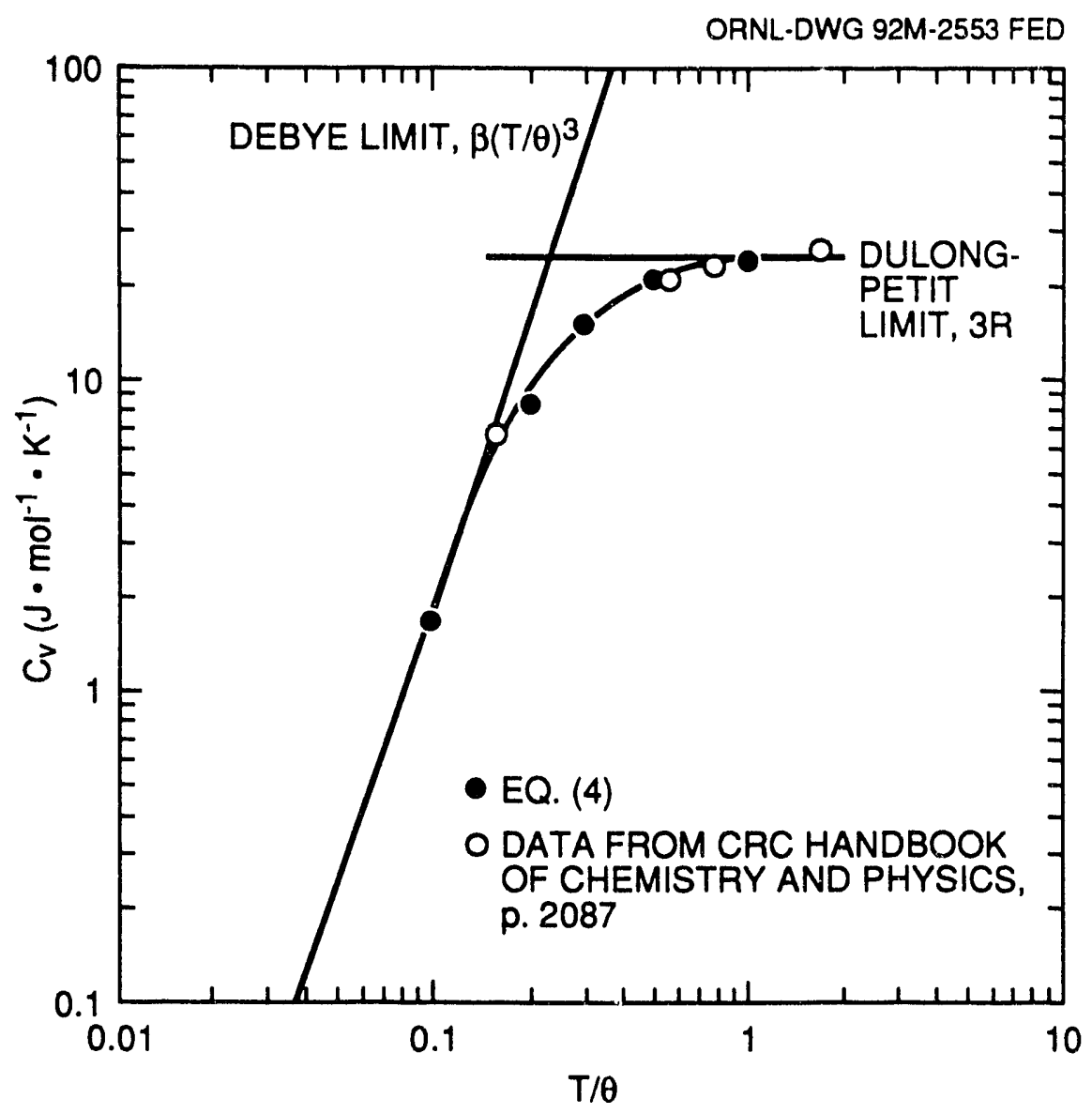

Fig. 3. The specific heat of silver $C_{\mathrm{v}}$ plotted vs reduced temperature $T / \theta$.

$$
T » \theta \text { (Dulong-Petit), } C_{\mathrm{v} 2}=3 R=24.95 \mathrm{~J} \cdot \mathrm{mol}^{-1} \cdot \mathrm{K}^{-1} \text {. }
$$

A family of curves that spans these two extreme limits is

$$
C_{\mathrm{v}}=\left(C_{\mathrm{v} 1}^{-n}+C_{\mathrm{v} 2}^{-n}\right)^{-1 / n} .
$$

As one can see from the figure, the value $n=0.85$ gives quite a good fit to the exact curve and is a useful approximation for computer calculation.

According to Reed and Clark, ${ }^{6} \theta=220 \mathrm{~K}$ for silver. The electronic term, which only plays a significant role at low temperatures, has the form

$$
C_{\mathrm{v}}=\gamma T,
$$

where $\gamma$, the so-called Sommerfeld constant, has the value $6.44 \times 10^{-4} \mathrm{~J} \cdot \mathrm{mol}^{-1} \cdot \mathrm{K}^{-2}$ (see ref. 7). 
Shown also in Fig. 3 are several experimental values of the specific heat of silver from the Handbook of Chemistry and Physics. ${ }^{8}$ The agreement with the curve calculated using Eqs. (4) and (5) is excellent.

\section{THE SPECIFIC HEAT OF BSCCO}

The specific heat of BSCCO materials has been reported by Mori et al., ${ }^{9}$ by Fisher et al., 10 and by Sasaki et al. ${ }^{10}$ According to Mori et al.,

$$
\begin{array}{ll}
C=\gamma T+\beta T^{3}, & 5 \mathrm{~K}<T<11 \mathrm{~K}, \\
\gamma=0.0095 \mathrm{~J} \cdot \mathrm{mol}^{-1} \cdot \mathrm{K}^{-2} ; \beta=0.00348 \mathrm{~J} \cdot \mathrm{mol}^{-1} \cdot \mathrm{K}^{-4} .
\end{array}
$$

The value of $\beta$ given here corresponds to the Debye value $r\left(1944 / \theta^{3}\right)$, where $\theta$, the Debye temperature of the BSCCO material, is $220 \mathrm{~K}$ and $r$, the number of atoms per molecule, is 19. (The material then has the composition $\mathrm{Bi}_{1.4} \mathrm{~Pb}_{0.6} \mathrm{Sr}_{2} \mathrm{Ca}_{2} \mathrm{Cu}_{3} \mathrm{O}_{10}$.) Note that the Debye temperature is the same as that of silver.

With the composition given here, the molecular weight $A$ is $1.023 \mathrm{~kg} / \mathrm{mol}$.

Collings ${ }^{10}$ gives the density $\rho_{d}$ as $6500 \mathrm{~kg} / \mathrm{m}^{3}$. Thus multiplying by $\rho_{d} / A$, we find

$$
\gamma_{\mathrm{BSCCO}}=60.36 \mathrm{~J} \cdot \mathrm{m}^{-3} \cdot \mathrm{K}^{-2} ; \beta_{\mathrm{BSCCO}}=22.11 \mathrm{~J} \cdot \mathrm{m}^{-3} \cdot \mathrm{K}^{-4} \text {. }
$$

Shown in Fig. 4 are the specific heats reported by Sasaki et al. ${ }^{10}$ [curve (a)] and Fisher et al. ${ }^{10}$ [curve (b)] compared with values calculated from the formulas (6) [curve (c)]. The agreement is quite good, so we shall continue to use the latter formulas.

The corresponding values of $\gamma$ and $\beta$ for silver are

$$
\gamma_{\mathrm{Ag}}=62.69 \mathrm{~J} \cdot \mathrm{m}^{-3} \cdot \mathrm{K}^{-2} ; \beta_{\mathrm{Ag}}=17.77 \mathrm{~J} \cdot \mathrm{m}^{-3} \cdot \mathrm{K}^{-4}
$$

\section{THE RESISTIVITY OF SILVER}

According to Matthiesen's rule, the resistivity of silver can be expressed as the sum of the temperature-independent residual resistivity $\rho_{r}$ and the temperature-dependent phonon resistivity $\rho_{\mathrm{p}}$. The latter is given, according to the Bloch-Grüneisen theory, by the equation

$$
y \equiv \frac{\theta \rho_{\mathrm{p}}}{K}=\frac{5}{4 z}\left(\frac{2}{z^{4}} \int_{0}^{z} \frac{t^{4}}{e^{t}-1} d t\right)-\frac{1}{e^{z}-1}, z=\frac{1}{x}=\frac{\theta}{T},
$$




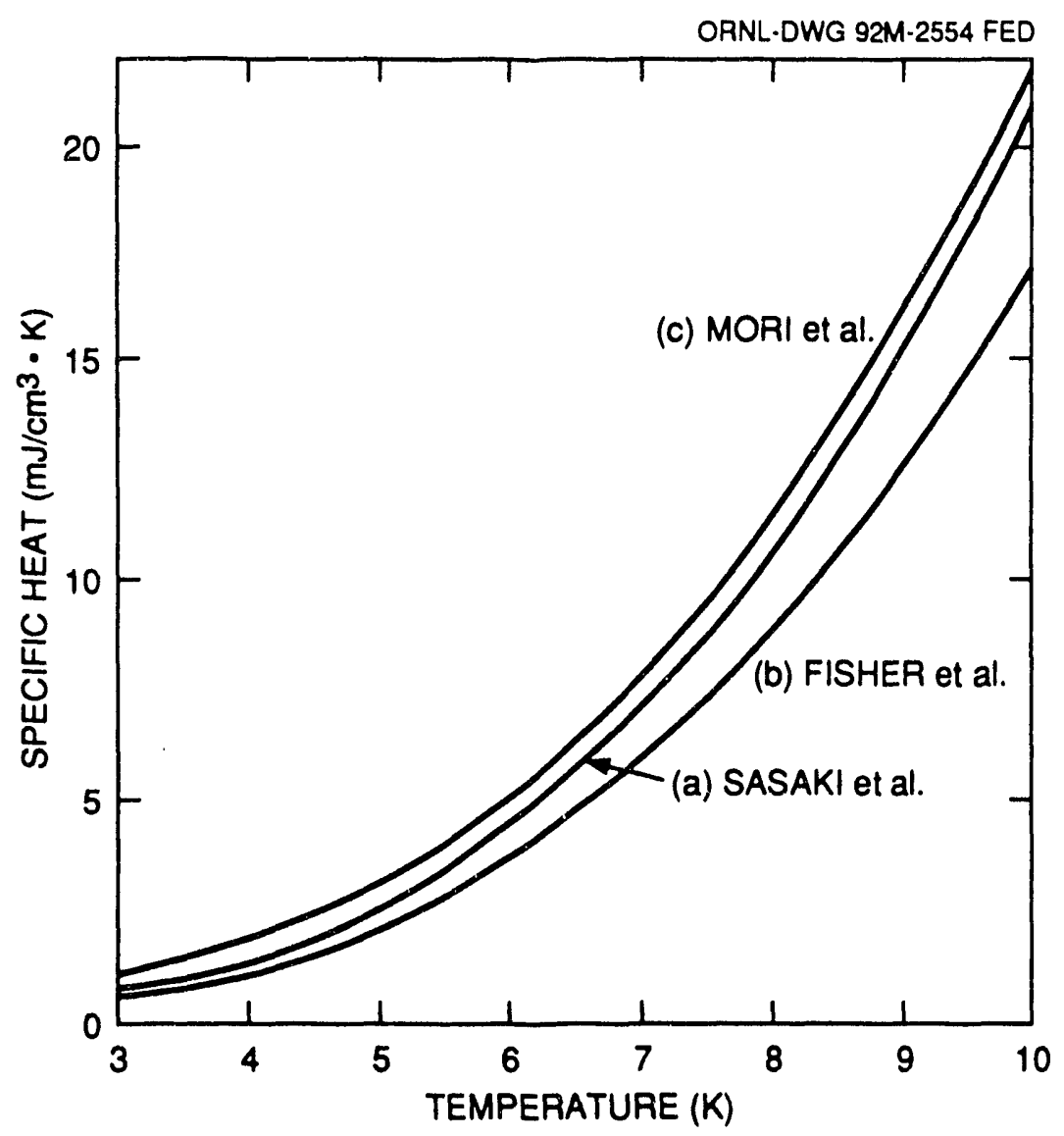

Fig. 4. The specific heat of BSCCO reported by (a) Sasaki et al., (b) Fisher et al., and (c) Mori et al.

where $K$ is a constant yet to be determined. Again the quantity in parentheses has been tabulated by Abramowitz and Stegun. 5

Shown in Fig. 5 is the quantity $y$ plotted against $x$ as calculated by means of Eq. (9). Shown also are the two limits

$$
\begin{array}{ll}
T \ll \theta, & y_{1}=124.4 x^{5}, \\
T » \theta, & y_{2}=x / 4 .
\end{array}
$$

The curve

$$
y=\left(y_{1}^{-n}+y_{2}^{-n}\right)^{-1 / n}, n=0.65
$$

fits the exact curve quite weil and will be used henceforth for the phonon resistivity. 


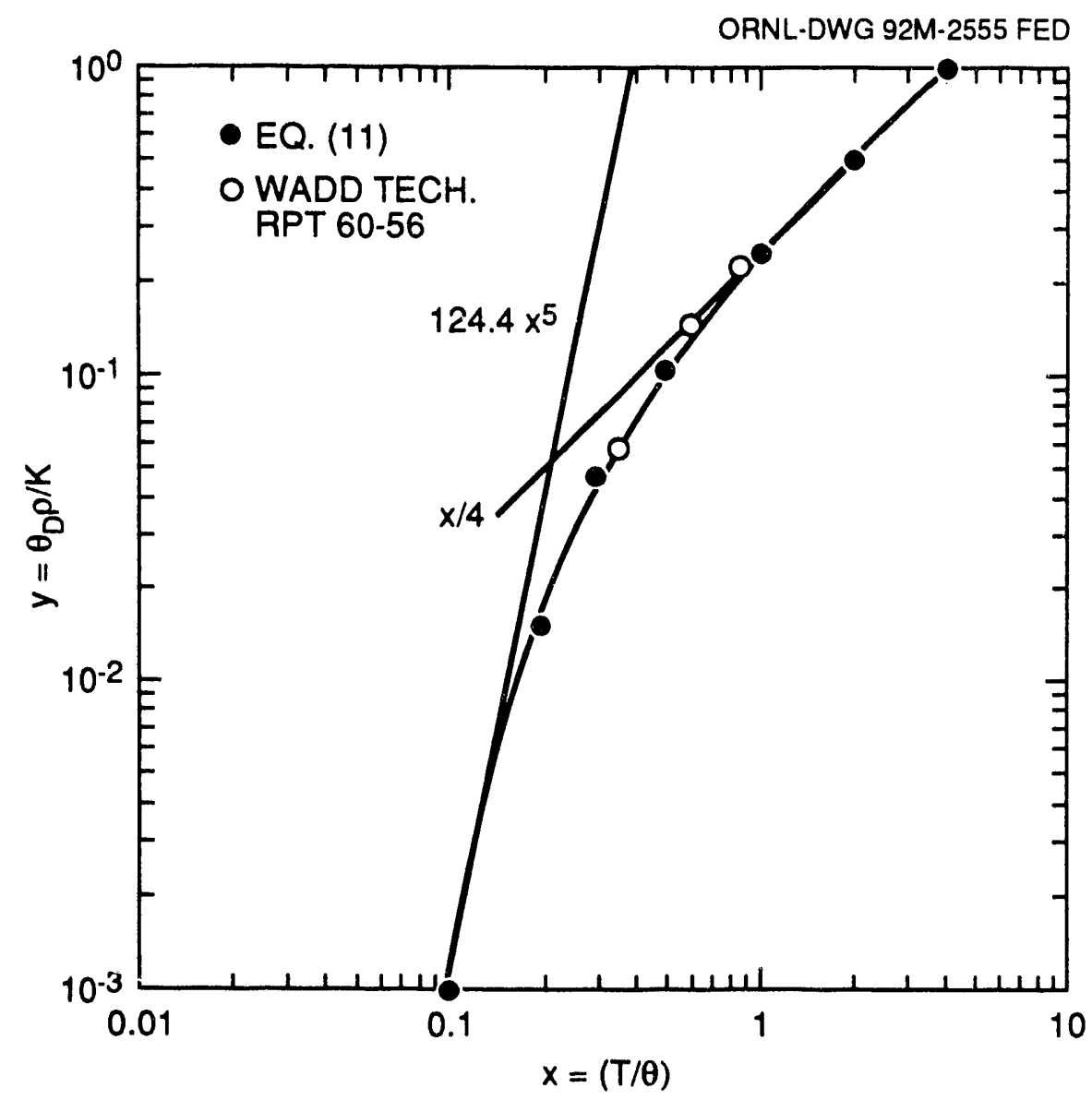

Fig. 5. The reduced phonon resistivity $\theta_{0} \rho / \bar{K}$ of silver plotted vs reduced temperature $T / \theta$.

Since the ice-point $(273 \mathrm{~K}$ ) resistivity of silver is $1.48 \mu \Omega \cdot \mathrm{cm}$ (ref. 6, Table 5.1, p. 165$), K=1.07 \times 10^{-5} \Omega \cdot \mathrm{m} \cdot \mathrm{K}$, and thus the phonon resistivity of silver is

$$
\rho_{\mathrm{Ag}, \mathrm{p}}=\left(4.845 \times 10^{-8}\right) y \Omega \cdot \mathrm{m} \text {. }
$$

For high enough temperatures (e.g., $>50 \mathrm{~K}$ ) the phonon resistivity is the dominant contribution to the resistivity. Also shown in Fig. 5 are measured values of the resistivity of silver. ${ }^{11}$ Their agreemient with the curve calculated from the Bloch-Grüneisen theory is excellent.

\section{THE HOT-SPOT FUNCTION}

A simple adiabatic heat balance shows that the hot-spot temperature $T_{\mathrm{hs}}$ is given by 


$$
\int_{T_{\mathrm{b}}}^{T_{\mathrm{hs}}} \frac{S}{\rho} d T=\int_{0}^{\infty} J^{2} d t
$$

where $S$ is the heat capacity per unit volume $\left(\mathrm{J} \cdot \mathrm{m}^{-3} \cdot \mathrm{K}^{-1}\right)$ and $\rho$ is the resistivity of the conductor.

Because the Debye temperatures of silver and BSCCO are equal (a lucky but useful coincidence), their phonon specific heats are in the ratio of their $\beta$ 's, namely, $\beta_{\mathrm{BSCCO}} / \beta_{\mathrm{Ag}}=1.244$. Above $20 \mathrm{~K}$, the Sommerfeld term $\gamma T$ makes a very small contribution $(<1 \%)$ to the total specific heat; and since $\gamma_{\mathrm{BSCCO}} / \gamma_{\mathrm{Ag}}=0.963$ is within $30 \%$ of the value of $\beta_{\mathrm{BSCCO}} / \beta_{\mathrm{Ag}}$ anyway, it is quite acceptable to take the ratio of the total specific heats to be $\beta_{\mathrm{BSCCO}} / \beta_{\mathrm{Ag}}$. Then for a $\mathrm{Ag} / \mathrm{BSCCO}$ composite with a volume fraction $f$ of silver and $1-f$ of $\mathrm{BSCCO}$,

$$
S=S_{\mathrm{Ag}}\left[f+(1-f)\left(\beta_{\mathrm{BSCCO}} / \beta_{\mathrm{Ag}}\right)\right]
$$

and

$$
\rho=\rho_{\mathrm{Ag}} / f
$$

so that

$$
\frac{S}{\rho}=\frac{S_{\mathrm{Ag}}}{\rho_{\mathrm{Ag}}} f\left[f+(1-f)\left(\beta_{\mathrm{BSCCO}} / \beta_{\mathrm{Ag}}\right)\right] .
$$

The second factor on the right-hand side Eq. (14c) is a simple multiplier to be applied to the hot-spot function of pure silver, which is shown in Fig. 6 as a function of $T_{\mathrm{hs}}$ for $T_{\mathrm{b}}=$ $20 \mathrm{~K}$ and three values of the residual resistance ratio, RRR, namely 100,500 , and 2500 .

\section{PROPAGATION VELOCITY}

In the range of temperatures $20-40 \mathrm{~K}$, the conductor will be cooled by stagnant gas (if it is cooled at all, i.e., if it is not potted). Such cooling is far less efficient than cooling by boiling helium, which is used with low-temperature superconductors. For this reason, we shall ignore cooling altogether. On the other hand, we cannot ignore the temperature dependences of the volumetric heat capacity $S$, the thermal conductivity $k$, and the resistivity $\rho$, as is often done when the propagation velocity of low-temperature superconductors is calculated. To facilitate the calculation, we shall take $S=\beta T^{3}$ and assume that $k$ and $\rho$ obey the Wiedemann-Franz law $k \rho=L_{0} T$.

The heat balance equation for a unit volume of conductor is then

$$
S \frac{\partial T}{\partial t}=\frac{\partial}{\partial z}\left(k \frac{\partial T}{\partial z}\right)+\rho J^{2} g(T)
$$




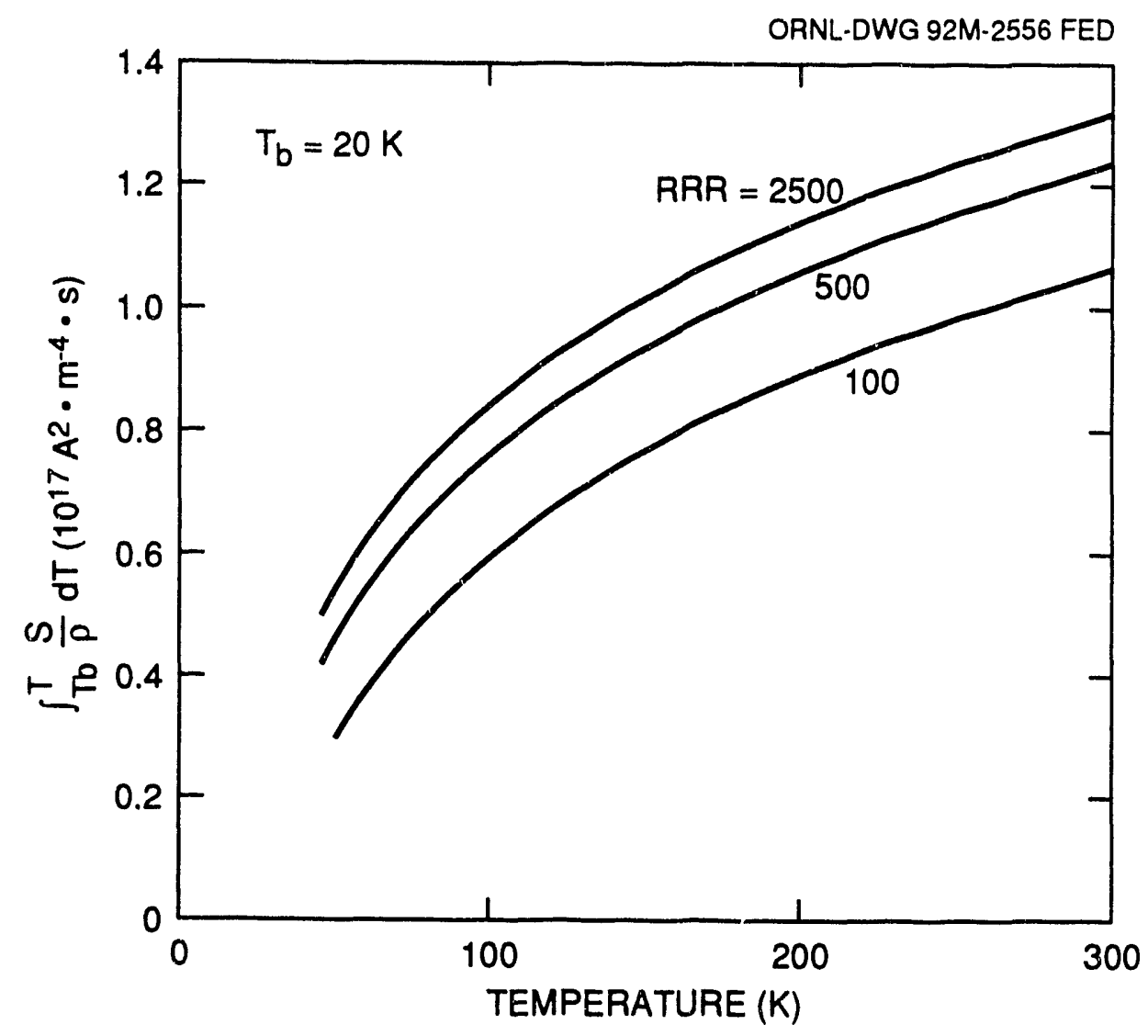

Fig. 6. The adiabatic hot-spot function for silver as a function of temperature with residual resistance ratio (RRR) as parameter.

where

$$
g(T)=\left\{\begin{array}{ll}
0, & T<T_{\mathrm{cs}} \equiv T_{\mathrm{b}}+(1-i)\left(T_{\mathrm{c}}-T_{\mathrm{b}}\right) \\
\frac{T-T_{\mathrm{cs}}}{T_{\mathrm{c}}-T_{\mathrm{cs}}}, & T_{\mathrm{cs}}<T<T_{\mathrm{c}} \\
1, & T>T_{\mathrm{c}}
\end{array} .\right.
$$

We look for a traveling-wave solution of Eq. (15), i.e., a solution of the form

$$
T=T(x+v t),
$$

which represents a wave traveling from right to left with a constant velocity $v$ (see Fig. 7). Substituting Eq. (17) into Eq. (15), we find 


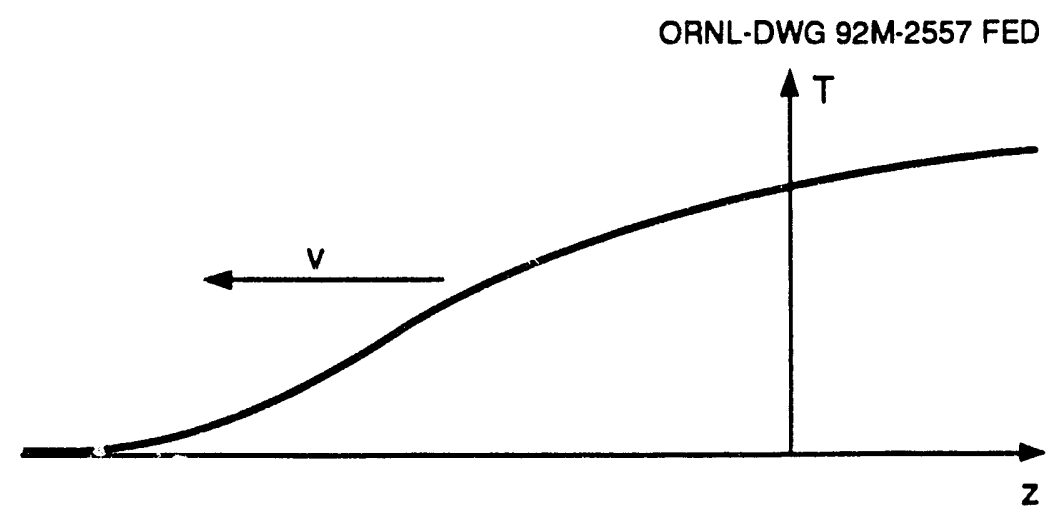

Fig. 7. Sketch of a temperature wave traveling from right to left.

$$
\frac{d}{d \xi}\left(k \frac{d T}{d \xi}\right)-v S \frac{d T}{d \xi}+\rho J^{2} g(T)=0
$$

where $\xi=x+v t$.

If we introduce the new variable $s=k(d T / d \xi)$, we can rewrite Eq. (18) as

$$
s \frac{d s}{d T}-v S s+L_{0} T J^{2} g(T)=0 .
$$

Next we introduce the dimensionless variables

$$
\begin{aligned}
& x=T / T_{\mathrm{b}}, \\
& y=s / L_{0}^{1 / 2} T_{\mathrm{b}} J, \\
& c=\nu S_{\mathrm{b}} / L_{0}^{1 / 2} J,
\end{aligned}
$$

which allows us to write Eq. (19) as

$$
y \frac{d y}{d x}-c x^{3} y+x g(x)=0,
$$

where

$$
g(x)= \begin{cases}0, & x<x_{\mathrm{cs}} \equiv i+(1-i) x_{\mathrm{c}} \\ \frac{x-x_{\mathrm{cs}}}{x_{\mathrm{c}}-x_{\mathrm{cs}}}, & x_{\mathrm{cs}}<x<x_{\mathrm{c}} \\ 1, & x>x_{\mathrm{c}}\end{cases}
$$


Our mathematical problem now reduces to finding the eigenvalue $c$ as a function of $i$ and $x_{c}$.

When $x<x_{\mathrm{cs}}$ and $g=0$, Eq. (21) is easy to solve:

$$
y=\frac{c}{4}\left(x^{4}-1\right),
$$

which obeys the necessary requirement that $y(1)=0$, i.e., that $d T / d \xi=0$ when $T=T_{\mathrm{b}}$.

When $x>x_{\mathrm{c}}$, the situation is more complicated and has been analyzed previously by the author. ${ }^{12}$ Shown in Fig. 8 is a sketch of the direction field of Eq. (21) when $g=1$, i.e., when $x>x_{\mathrm{c}}$. The integral curves consist of two families separated by a separatrix. As $x \rightarrow \infty$, the curves of the upper family pass through a minimum. Since $y$ is proportional to the heat flux, these upper curves cannot describe the solution sketched in which the flux has a maximum, not a minimum. Neither can the curves of the lower family, for which $y$ changes sign, describe the solution sketched in Fig. 7. So by elimination we are left with the separatrix.

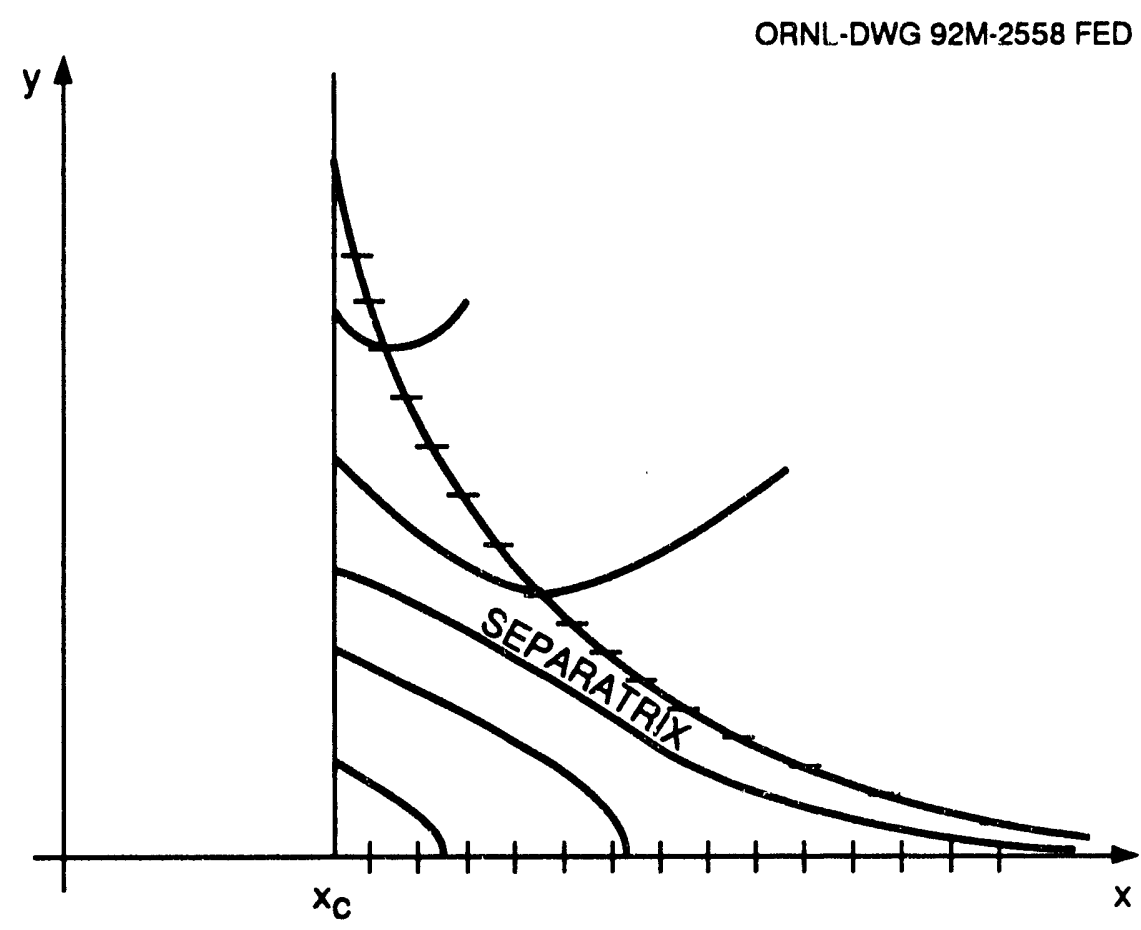

Fig. 8. The direction field of Eq. (21) when $g=1$, i.e., when $x>x_{\mathrm{c}}$. 
If we set $y^{\prime}=c^{1 / 3} y$ and $x^{\prime}=c^{1 / 3} x$ we find that

$$
y^{\prime} \frac{d y^{\prime}}{d x^{\prime}}-x^{\prime 3} y^{\prime}+x^{\prime}=0
$$

After a straightforward calculation, we find the following asymptotic series for the separatrix:

$$
y^{\prime}=\frac{1}{x^{\prime 2}}-\frac{2}{x^{\prime 8}}+\frac{20}{x^{\prime 14}}-\frac{352}{x^{\prime 20}}+\frac{8624}{x^{\prime 26}}-\frac{266784}{x^{\prime 32}}+\ldots
$$

The quantity $c$ is calculated in the following steps:

1. Choose $x_{\mathrm{c}}$ and $i\left(x_{\mathrm{c}}>1,0<i<1\right)$.

2. Guess $c$.

3. Set $x^{\prime}=c^{1 / 3} x$ and choose $x$ large enough to use Eq. (25) to calculate $y^{\prime}$.

4. Using $x$ and $y=y^{\prime} / c^{1 / 3}$ as starting values, integrate Eq. (21) numerically from $x_{\mathrm{c}}$ to $x_{\mathrm{cs}}$.

5. Calculat the difference $D(c)=y\left(x_{\mathrm{cs}}\right)-(c / 4)\left(x_{\mathrm{cs}}^{4}-1\right)$.

6. If $D(c) \neq 0$, return to step 2. Continue until $D(c)=0$.

Rapid convergence can be assured if the Newton-Raphson method is used to find the root of $D(c)$.

Figure 9 shows values of $c$ plotted vs $i$ for $x_{\mathrm{c}}=1.5$ and 2.0.

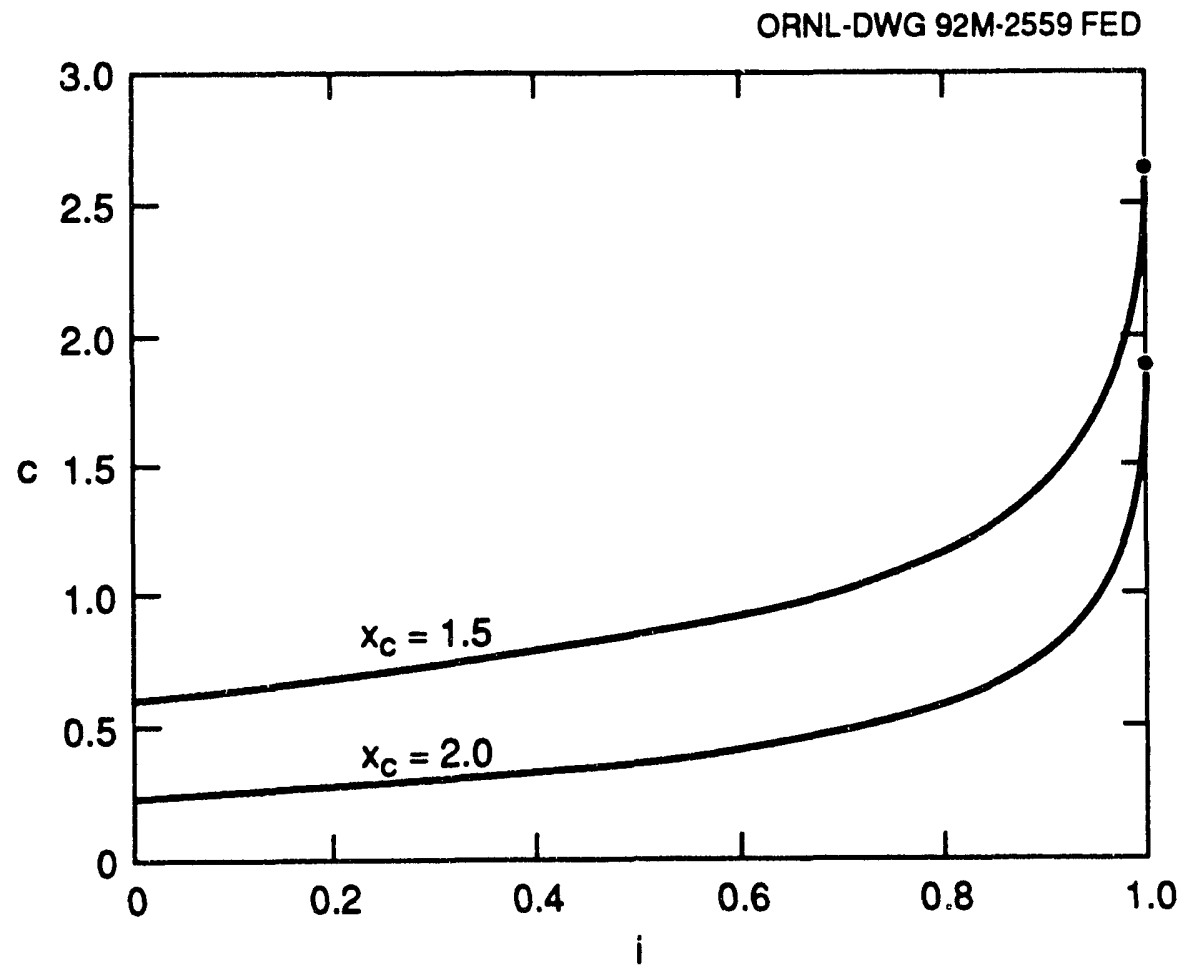

Fig. 9. The dimensionless velocity $c$ plotted vs $i$ for $x_{\mathrm{c}}=1.5$ and 2.0. 


\section{NORMAL-ZONE VOLTAGE}

We can use the theory just describ $\mathrm{d}$ to determine the normal-zone voltage as a function of the hot-spot temperature. Because of the sharp tise of resistivity with temperature, most of the voltage will be contributed hy the hot center of the normal zone, where $T_{\mathrm{hs}} » T_{\mathrm{c}}>T_{\mathrm{b}}$, i.e., where $x » 1$. Then we can use just the first term in Eq. (25) to describe the variation of $y$ with $x: y=1 / c x^{2}$. In dimensional terms this is

$$
s=\frac{L_{0}^{1 / 2} J T_{\mathrm{b}}^{3}}{c T^{2}} .
$$

Thus

$$
d \xi=\frac{k d T}{s}=\frac{k c T^{2}}{L_{0}^{1 / 2} J T_{\mathrm{b}}^{3}} d T
$$

At a fixed time, $d \xi$ is an increment of length of the normal zone and contributes an increment $d V$ to the total voltage:

$$
d V=J \rho d \xi=c L_{0}^{1 / 2}\left(\frac{T}{T_{\mathrm{b}}}\right)^{3} d T
$$

The total voltage $V$ is ther:

$$
V=\frac{1}{2} c L_{0}^{1 / 2} T_{\mathrm{b}}\left(\frac{T_{\mathrm{hs}}}{T_{\mathrm{b}}}\right)^{4},
$$

an extra factor of 2 coming from the fact that the normal zone expands in two directions.

When $T_{\mathrm{bs}}=100 \mathrm{~K}, T_{\mathrm{b}}=20 \mathrm{~K}$, and $c=0.373\left(x_{\mathrm{c}}=2, i=0.5\right), V=0.365 \mathrm{~V}$, which should be more than adequate for detection. If the $R R R=100$, the hot-spot function for the silver will be $5.90 \times 10^{16} \mathrm{~A}^{2} \cdot \mathrm{m}^{-4}$. $\mathrm{s}$. Since we have chosen $J_{\mathrm{c}}$ of the BSCCO to be $5 \mathrm{kA} / \mathrm{cm}^{2}, f=0.5$, and the correction factor of Eq. (14c) is then 0.561. The hot-spot function for the composite is then $3.31 \times 10^{16} \mathrm{~A}^{2} \cdot \mathrm{m}^{-4}$.s. With $J=2.5 \mathrm{kA} / \mathrm{cm}^{2}$, we find from Eq. (13) that $t=53.0 \mathrm{~s}$. According to Eqs. (3), (4), and (14a), $S_{\mathrm{b}}=1.44 \times$ $10^{5} \mathrm{~J} / \mathrm{m}^{3} \cdot \mathrm{K}$; then according to Eq. $(20 \mathrm{c}), v=1.01 \mathrm{~cm} / \mathrm{s}$. The normal zone is then $53.7 \mathrm{~cm}$ long when the hot-spot temperature reaches $100 \mathrm{~K}$.

\section{THERMAL STRESS}

Ochiai et al. ${ }^{13}$ have pointed out the following mechanism by which oxide superconductors may be damaged by warming up. When the reacted conductor is cooled 
down from the reaction temperature, the silver contracts more than the oxide, putting the silver in tension and the oxide in compression. The silver typically reaches its elastic limit in the early stages of cooldown, after which its tensile stress remains fixed at its yield stress $\sigma_{\mathrm{y}}$ during the remainder of cooldown (see Fig. 10 for the assumed stressstrain curve of the silver). Thus when cooldown is over, we find the conductor in a state in which the silver suffers a tensile stress $\sigma_{\mathrm{y}}$ and the oxide a compressive stress $-f_{1} \sigma_{\mathrm{y}} / f_{2}$, where $f_{1}$ is the volume fraction of silver and $f_{2}$ that of the oxide.

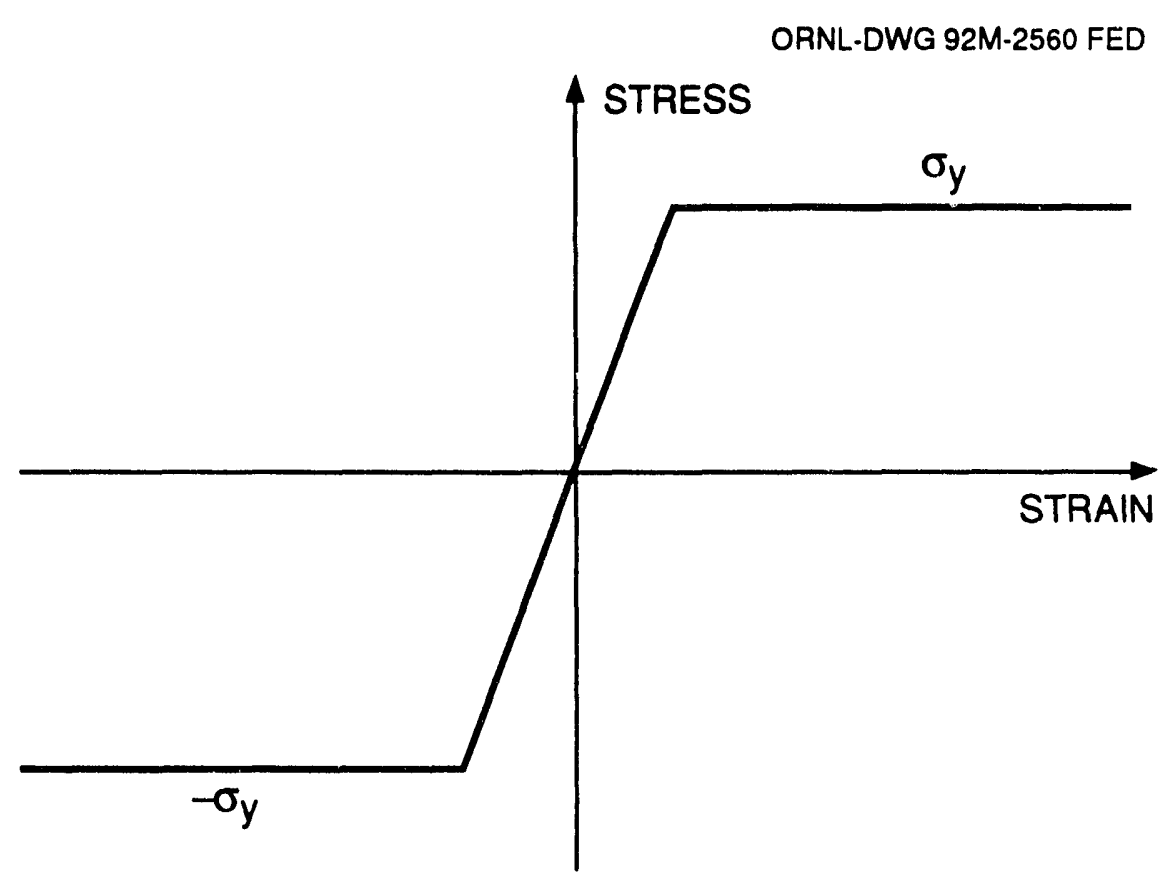

Fig. 10. The assumed stress-strain curve of silver.

If a normal zone is formed, the silver expands thermally more than the oxide, threatening to put the oxide in tension and the silver in compression. However, the maximum compressive stress that the silver can endure is its compressive yield stress $-\sigma_{y}$, so that the oxide experiences at most a tension $f_{1} \sigma_{y} / f_{2}$. Now as it happens, the yield stress of the annealed silver studied by Ochiai et al. is only $13 \mathrm{MPa}$, whereas the ultimate tensile strength of their BSCCO is roughly $60 \mathrm{MPa}$. Their tapes had $f_{1}=0.7$ and $f_{2}=0.3$ 
so that $f_{1} \sigma_{\mathrm{y}} / f_{2}=30.3 \mathrm{MPa}$. BSCCO conductors should be able to stand this tension. So the thermal stresses caused by the first quench should do no harm.

We may decide to limit the hot-spot temperature so that the compressive stress in the silver induced by thermal expansion never exceeds $\sigma_{y}$. Then the silver does not leave the elastic region during a quench and so never work hardens. In such a case, the thermal stresses caused by subsequent quenches should also do no harm.

Consider the conductor sketched in Fig. 11. The stresses entered in the various regions are those that exist after cooldown. If we reckon strains based on material displacements from this configuration, the stress-strain curve of the silver is then that of Fig. 12. We take the stress-strain curve of the BSCCO to be entirely elastic:

$$
\sigma_{2}=E_{2} \eta_{2}-\frac{f_{1} \sigma_{y}}{f_{2}}
$$

The force-free condition for the conductor is

$$
f_{1} \sigma_{1}+f_{2} \sigma_{2}=0
$$

ORNL-DWG 92M-2561 FED

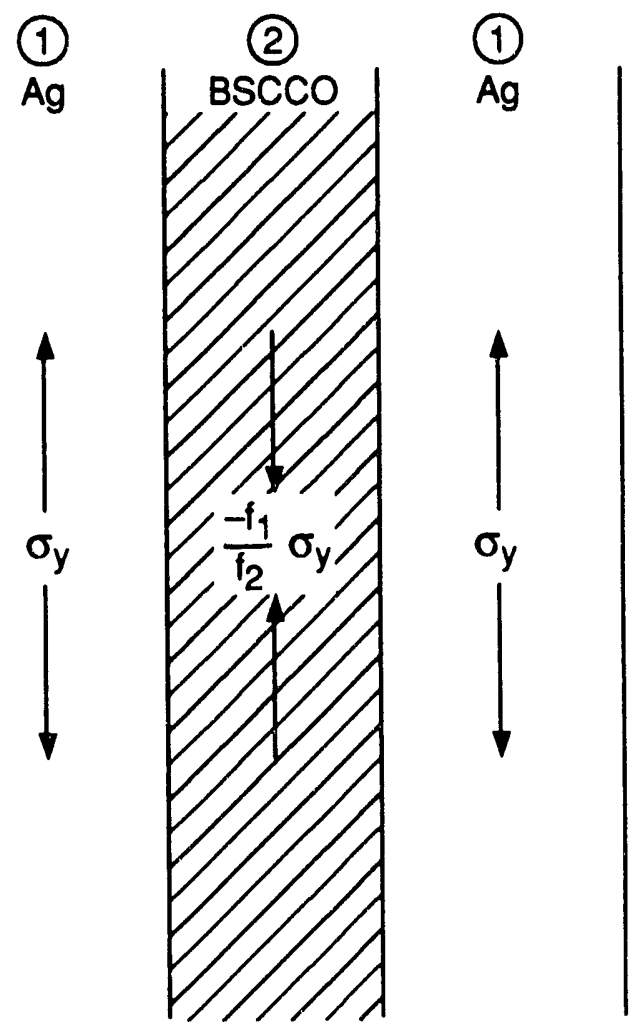

Fig. 11. A schematic depiction of the state of stress in a $\mathrm{Ag} / \mathrm{BSCCO}$ conductor after cooldown. 
and the condition that the silver and the BSCCO have the same length is

$$
\eta_{1}+\alpha_{1} \theta=\eta_{2}+\alpha_{2} \theta
$$

where $\alpha_{1,2}$ are the linear coefficients of thermal expansion of silver and BSCCO, respectively, and $\theta$ is the temperature rise.

If we eliminate $\eta_{2}$ and $\sigma_{2}$ from Eq. (30) using Eqs. (31) and (32), we find

$$
\eta_{1}+\frac{f_{1}}{f_{2} E_{2}}\left(\sigma_{1}-\sigma_{y}\right)+\left(\alpha_{1}-\alpha_{2}\right) \theta=0
$$

The values of $\eta_{1}$ and $\sigma_{1}$ are determined by the intersection of the line (33) and the broken line of Fig. 12.

If the line (33) passes through the point $P:\left(-2 \sigma_{y} / E_{1},-\sigma_{y}\right)$, the temperature given by Eq. (33) will then be the maximum allowable that just avoids cold working the silver. Using the values $E_{1}=80 \mathrm{GPa}, E_{2}=54 \mathrm{GPa}, \alpha_{1}=2 \times 10^{-5} \mathrm{~K}^{-1}$, and $\alpha_{2}=8 \times 10^{-6} \mathrm{~K}^{-1}$ given by Ochiai et al., we find

ORNL-DWG 92M-2562 FED

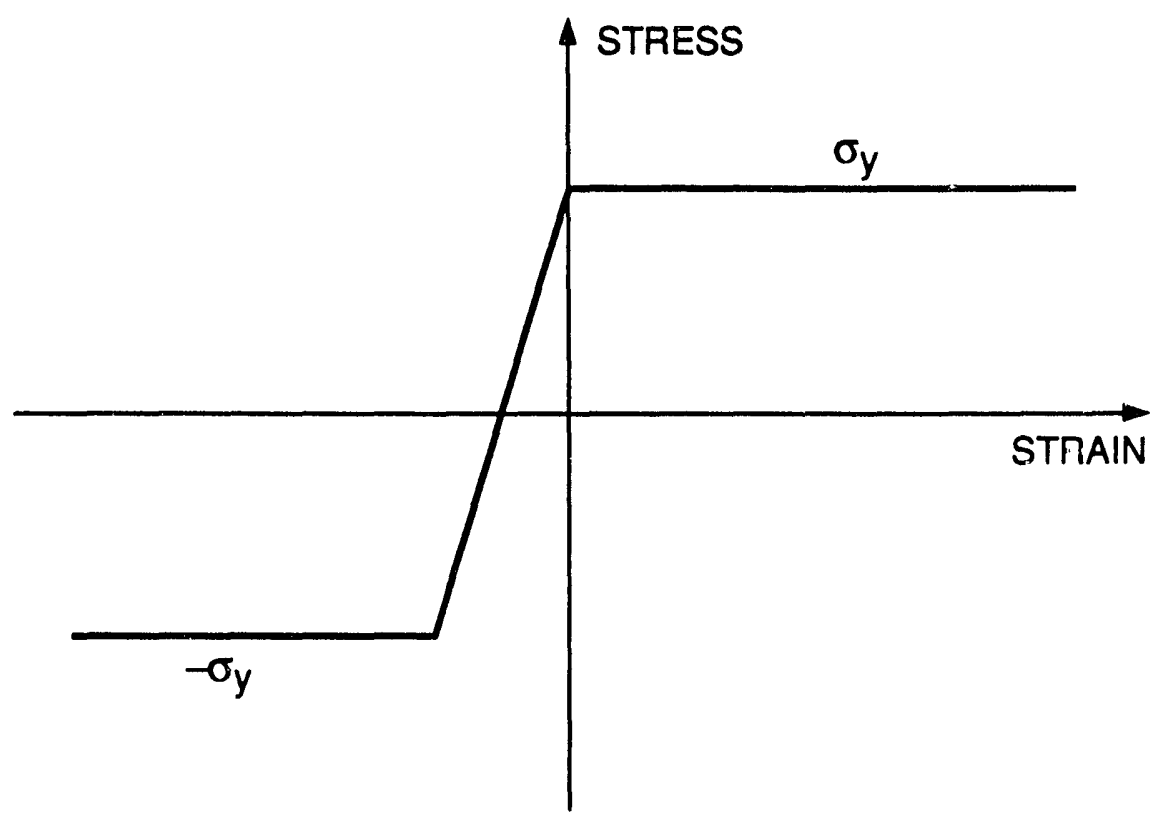

Fig. 12. The stress-strain curve of silver when the strains are reckoned based on material displacements from the configuration of Fig. 11. 


$$
\theta=-\frac{2 \sigma_{\mathrm{y}}}{\alpha_{1}-\alpha_{2}} \cdot \frac{f_{1} E_{1}+f_{2} E_{2}}{f_{2} E_{1} E_{2}}=121 \mathrm{~K}
$$

If the line (33) passes through the point $Q:\left(-\sigma_{\mathrm{y}} / E_{1}, 0\right)$, the BSCCO will never go into tension at all. The temperature rise will then be half the value given by Eq. (34), namely, $60 \mathrm{~K}$. Adding this to the assumed onerating temperature of $20 \mathrm{~K}$, we find $80 \mathrm{~K}$ for the maximum hot-spot temperature that never puts the BSCCO into tension. This is consistent with the $100 \mathrm{~K}$ hot-spot temperature used for estimation in the previous sections.

\section{QUENCH ENERGY, PART 1}

As mentioned in the introduction, our task is to study the protection of the magnet, presupposing that a propagating normal zone has somehow been established. For completeness, we study here the likelihood of such an event by evaluating the quench energy (defined as the smallest, sudden energy deposition at a point that still causes a quench).

The quench energies of conventional potted magnets that operate at $4.2 \mathrm{~K}$ with millimeter-size conductors are a few to a few tens of millijoules. The main cause of quenches in such magnets is cracking of the epoxy potting caused by the Lorentz force. Acoustic emission studies have shown that some, but not all, instances of cracking cause quenching. We may infer from this that the energies released when the epoxy cracks are distributed from a few to a few tens of millijoules, sometimes exceeding the quench energy and sometimes not.

As we shall see below, the quench energies of potted Ag/BSCCO magnets that operate in the temperature range $20-40 \mathrm{~K}$ with millimeter-size conductors are several orders of magnitude greater than those of their conventional counterparts. Therefore, we expect the Ag/BSCCO magnets to be immune to the effects of epoxy cracking. In other words, we expect them not to train.

To calculate the quench energy, we again start with the one-dimensional heat balance equation, Eq. (15). Imagine a heat pulse delivered suddenly at time $t=0$ in the plane $z=0$; its areal density in that plane is $Q\left(\mathrm{~J} / \mathrm{m}^{2}\right)$. When $Q$ is small, the conductor temperature eventually falls below the current-sharing threshold and the magnet recovers. When $Q$ is large, the conductor temperature eventually increases without bound and the magnet quenches. We seek the value of $Q$ that separates these two possibilities.

The parameters that appear in Eq. (15) are $S\left(\mathrm{~J} / \mathrm{m}^{3} \cdot \mathrm{K}\right), k(\mathrm{~W} / \mathrm{m} \cdot \mathrm{K})$, and $\rho J^{2}\left(\mathrm{~W} / \mathrm{m}^{3}\right)$. Implicit in the definition of the function $g(T)$ are the three temperatures $T_{\mathrm{b}}, T_{\mathrm{cs}}$, and $T_{\mathrm{c}}$. Dimensional analysis shows that $Q$ must be related to these parameters in the following way: 


$$
Q=S T\left(\frac{k T}{\rho J^{2}}\right)^{1 / 2} F\left(\frac{T_{\mathrm{b}}}{T_{\mathrm{c}}}, \frac{T_{\mathrm{cs}}}{T_{\mathrm{c}}}\right)
$$

Here no subscript appears on $T$ because an equation of the form (35a) holcs whether $T$ is $T_{\mathrm{b}}, T_{\mathrm{cs}}, T_{\mathrm{c}}$, or any other temperature; however, the form and value of the unknown function $F$ vary according to the definition $T$. If we assume as we did earlier that $k$ and $\rho$ for silver obey the Wiedemann-Franz law, Eq. (35a) becomes

$$
Q=\frac{S T^{2} L_{0}^{1 / 2}}{\rho J} F\left(\frac{T_{\mathrm{b}}}{T_{\mathrm{c}}}, \frac{T_{\mathrm{cs}}}{T_{\mathrm{c}}}\right)
$$

Plutted in Fig. 13 is the parameter cluster $S T 2 / \rho$ for silver as a function of temperature for $R R R=100,500$, and 2500 . In the temperature range from 20 to $40 \mathrm{~K}$, it is within a factor of 2 of $10^{18} \mathrm{~A}^{2} \cdot \mathrm{m}^{-4} \cdot \mathrm{s} \cdot \mathrm{K}$, so that the yalue of $Q$ does not depend critically on the choice of the temperature $T$. The value of $S / \rho$ needs to be corrected as shown in Eq. (14c), but the correction factor is close to 1 and is ignored here. We also ignore the packing fraction of the conductor in calculating its current density, which we take to be equal to the overall current density, $2.5 \times 10^{7} \mathrm{~A} / \mathrm{m}^{2}$. Then the first factor in Eq. (35b) is roughly $6 \times 10^{6} \mathrm{~J} / \mathrm{m}^{2}=6 \mathrm{~J} / \mathrm{mm}^{2}$. Thus for a $1-\mathrm{mm}$-diam wire, the quench energy (excluding the second factor $F$ ) is about $5 \mathrm{~J}$.

\section{QUENCH ENERGY, PART 2}

To refine this order-of-magnitude estimate, we must calculate the function $F$. This calculation may be rough; it suffices only to gage the order of magnitude of $Q$. To facilitate the calculation, we take as before $S=\beta T^{3}$ and $k \rho=L_{0} T$, the Wiedemann-Franz law. In the range $20-40 \mathrm{~K}, \rho$ also varies closely with the third power of $T$ for RRR = 100,500 , and 2500 . So we take

$$
\rho=\rho_{*} T^{3}, k=k_{*} T^{-2}, k_{*} \rho_{*}=L_{0}
$$

The parameters entering the problem with their units are then

$$
\begin{gathered}
\beta \mathrm{J} \cdot \mathrm{m}^{-3} \cdot \mathrm{K}^{-4}, \\
k_{*} \mathrm{~W} \cdot \mathrm{m}^{-1} \cdot \mathrm{K}, \\
\rho_{*} \Omega^{2} \mathrm{~W} \cdot \mathrm{m}^{-3} \cdot \mathrm{K}^{-3}, \\
Q \mathrm{~J} \cdot \mathrm{m}^{-2} .
\end{gathered}
$$




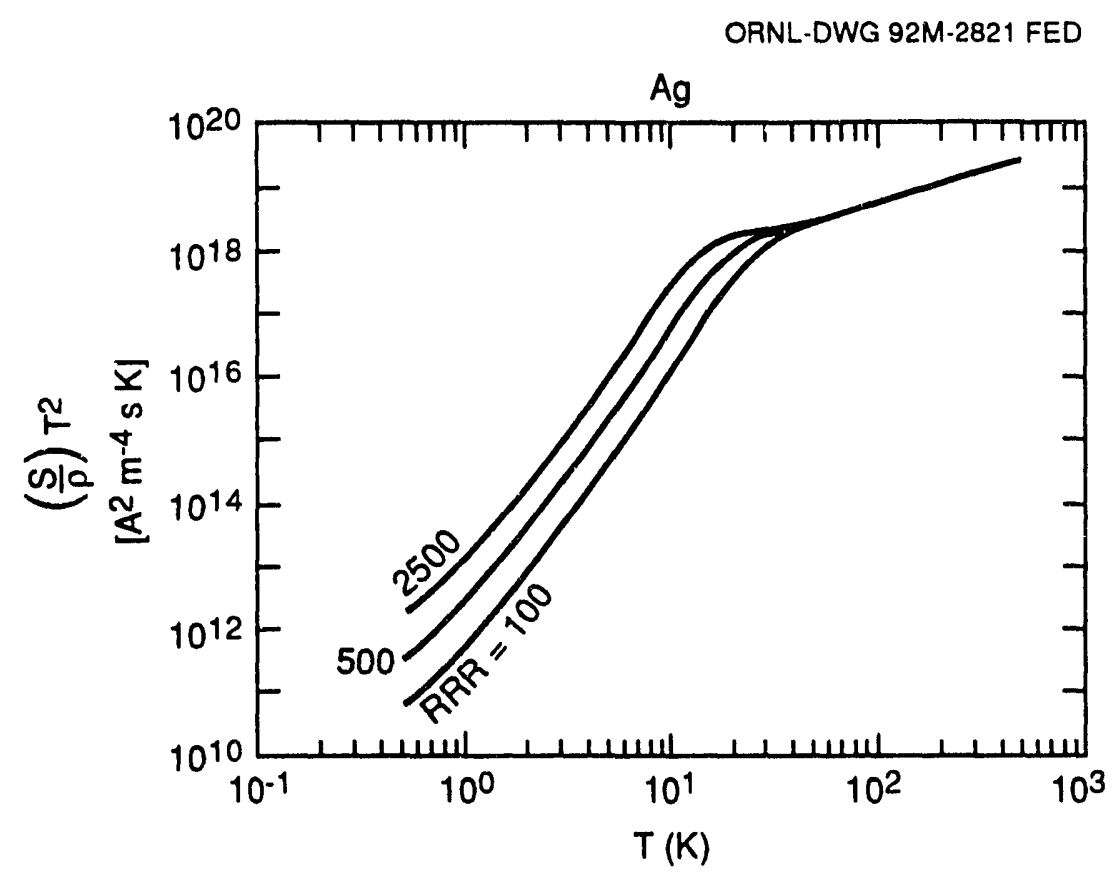

Fig. 13. The parameter cluster $S T 2 / \rho$ as a function of temperature with residual resistivity as parameter.

There is no dimensionless combination of these quantities, so we can choose a special set of units in which they are all numerically equal to 1 . Then Eq. (15) becomes

$$
T^{3} \frac{\partial T}{\partial t}=\frac{\partial}{\partial z}\left(T^{-2} \frac{\partial T}{\partial z}\right)+T^{3} g(T) .
$$

For very short times after the initial pulse, we can ignore the heat production term $g(T)$. Then Eq. (38) becomes

$$
T^{3} \frac{\partial T}{\partial t}=\frac{\partial}{\partial z}\left(T^{-2} \frac{\partial T}{\partial z}\right) .
$$

The solution of Eq. (39) then describes the form of the solution of Eq. (38) directly after the pulse. The solution we seek is determined by the boundary and initial conditions

$$
T( \pm \infty, t)=T_{\mathrm{b}}, T(z, 0)=T_{\mathrm{b}},
$$


and the heat conservation condition

$$
\begin{gathered}
\int_{-\infty}^{\infty}\left(\frac{\beta T^{4}}{4}-\frac{\beta T_{\mathrm{b}}^{4}}{4}\right) d z=Q \quad \text { (ordinary units) } \\
\int_{-\infty}^{+\infty}\left(T^{4}-T_{\mathrm{b}}^{4}\right) d z=4 \quad \text { (special units) }
\end{gathered}
$$

Since for the moment we are interested in the early stages of heat conduction, in which $T(0, t) » T_{\mathrm{b}}$, we can set $T_{\mathrm{b}}=0$ without seriously compromising the calculation of $T(0, t)$, which is the temperature that determines the issue of recovery or quench. When $T_{b}=0$, Eqs. (39) and (40) have the following solution:

$$
T=t^{-1 / 3} y\left(z / t^{4 / 3}\right)
$$

where

$$
y(x)=\left(\frac{6}{5}\right)^{1 / 5}\left(x^{2}+a^{2}\right)^{-1 / 5}
$$

and

$$
a=\left(\frac{6}{5}\right)^{4 / 3}\left[\frac{\sqrt{\pi}}{4} \frac{\Gamma(3 / 10)}{\Gamma(4 / 5)}\right]^{5 / 3}=1.583192808 \ldots
$$

For later times, when we can no longer ignore $g(T)$, we can try to approximate the solution to Eq. (38) as

$$
T(z, t)=h(t) T_{0}(z, t)
$$

where $h(t)$ is a function yet to be determined and $T_{0}(z, t)$ is the solution given in Eq. (41). If we substitute Eq. (42) into Eq. (38), we find

$$
\dot{h} T_{0}^{4}+h T_{0}^{3} \frac{\partial T_{0}}{\partial t}=h^{-4} \frac{\partial}{\partial z}\left(T_{0}^{-2} \frac{\partial T_{0}}{\partial z}\right)+T_{0}^{3} g\left(h T_{0}\right)
$$

Now because Eq. (42) is not the exact form of the solution (however good an approximation it may be), no choice of $h(t)$ exactly satisfies Eq. (43). But we can find an $h(t)$ that satisfies it in an average sense, for when we integrate Eq. (43) over $z$ from $-\infty$ to $+\infty$, we find the equation 


$$
4 \dot{h}=\int_{-\infty}^{\infty} T_{0}^{3} g\left(h T_{0}\right) d z
$$

to which we can find a solution $h(t)$.

If in Eq. (44) we replace $g(T)$ by the form it has in the current sharing region, namely $\left.\left(T-T_{\mathrm{cs}}\right) / T_{\mathrm{c}}-T_{\mathrm{cs}}\right)$, we can solve Eq. (44) explicitly. By making this replacement, we make the conductor less stable because we imply $g(T)>1$ for $T>T_{\mathrm{c}}$ and $g(T)<0$ for $T<T_{\mathrm{cs}}$. Thus our final estimate for $Q$ is an underestimate. Equation (44) now becomes

$$
\begin{aligned}
4 \dot{h} & =\int_{-\infty}^{+\infty} T_{0}^{3}\left(\frac{h T_{0}-T_{\mathrm{cs}}}{T_{\mathrm{c}}-T_{\mathrm{cs}}}\right) d z \\
& =\frac{4}{T_{\mathrm{c}}-T_{\mathrm{cs}}} h-\frac{T_{\mathrm{cs}}}{T_{\mathrm{c}}-T_{\mathrm{cs}}}-\int_{-\infty}^{+\infty} T_{0}^{3} d z
\end{aligned}
$$

Now

$$
\int_{-\infty}^{+\infty} T_{0}^{3} d z=t^{1 / 3} \int_{-\infty}^{+\infty} y^{3} d x=4 b t^{1 / 3}
$$

where

$$
b=\left(\frac{6}{5}\right)^{3 / 5} a^{-1 / 5} \frac{\sqrt{\pi}}{4} \frac{\Gamma(1 / 10)}{\Gamma(3 / 5)}=2.880758410 \ldots
$$

Therefore

$$
\dot{h}-\frac{h}{T_{\mathrm{c}}-T_{\mathrm{cs}}}=-\left(\frac{T_{\mathrm{cs}}}{T_{\mathrm{c}}-T_{\mathrm{cs}}}\right) b t^{1 / 3}
$$

We must solve Eq. (47) subject to the initial condition $h(0)=1$. The solution we seek is

$$
h=\left[1-\Gamma(4 / 3) b T_{\mathrm{cs}}\left(T_{\mathrm{c}}-T_{\mathrm{cs}}\right)^{1 / 3}+\frac{b T_{\mathrm{cs}}}{T_{\mathrm{c}}-T_{\mathrm{cs}}} \int_{t}^{\infty} t^{1 / 3} e^{-t /\left(T_{\mathrm{c}}-T_{\mathrm{cs}}\right)} d t\right] e^{t /\left(T_{\mathrm{c}}-T_{\mathrm{cs}}\right)} .
$$

If the sum of the first two terms in Eq. (48) is $>0, h$ increases asymptotically without bound, rising exponentially with time. If the sum of these two terms is $<0, h$ decreases asymptotically without bound. These two regions of behavior are separated by the 
vanishing of the first two terms in Eq. (48), which is the bifurcation condition we have been seeking.

The bifurcation condition can be writren

$$
T_{\mathrm{cs}}\left(T_{\mathrm{c}}-T_{\mathrm{cs}}\right)^{1 / 3}=\frac{1}{b \Gamma(4 / 3)}=0.388733230 \ldots \equiv C
$$

in special units. To reconstitute this equation into ordinary units, we must supply where needed appropriate powers of the quantities in Eqs. (37) in order to make it dimensionally hoinogeneous. This can be done in only one way, the result of which is

$$
Q=C^{-3 / 2} \beta\left(\frac{k_{*}}{\rho_{*} J^{2}}\right)^{1 / 2} T_{\mathrm{cs}}^{3 / 2}\left(T_{\mathrm{c}}-T_{\mathrm{cs}}\right)^{1 / 2}
$$

If we define $i=J / J_{\mathrm{c}}$ and remember that $T_{\mathrm{cs}}=T_{\mathrm{b}}+(1-i)\left(T_{\mathrm{c}}-T_{\mathrm{b}}\right)=(1-i) T_{\mathrm{c}}$ when $T_{\mathrm{b}}=$ 0 , we can rewrite Eq. (49b) as

$$
Q=\frac{S_{\mathrm{c}} T_{\mathrm{c}}^{2} L_{0}^{1 / 2}}{\rho_{\mathrm{c}} J_{\mathrm{c}}} \cdot \frac{C^{-3 / 2}(1-i)^{3 / 2}}{\sqrt{i}} .
$$

Comparing Eq. (49c) with Eq. (35b), we see that $F=[(1-i) / C]^{3 / 2} \sqrt{i}$. When $i=0.5, F=$ 1.031 , so that our earlier estimate of $Q$ hardly changes at all. When $i=0.9$, however, $F=$ 0.1238 , and our earlier estimate of $Q$ is substantially reduced. 


\section{REFERENCES}

1. H. Kumakura, K. Tagano, E. Yanagisawa, J.-I. Kase, and H. Maeda, Jpn. J. Appl. Phys. 29, L1652 (1990).

2. K. Aihara, M. Okada, T. Matsumoto, S. Maisuda, F. Hosono, and M. Seido, IEEE Trans. Magn. MAG-27, 894-6 (1991).

3. T. A. Miller, L. A. Schwarzkopf, J. E. Ostenson, D. K. Finnemore, J. Righi, R. A. Gleixner, and D. Ziegler, IEEE Trans. Magn. MAG-27, 909-11 (1991).

4. Superconductor Week, January 20, 1992, p. 3.

5. M. Abramowitz and I. A. Stegun, Handbook of Mathematical Functions, Dover, New York, 1965, p. 998.

6. R. P. Reed and A. F. Clark, eds., Materials at Low Temperature, American Society for Metals, Metals Park, Ohio, 1983, Table 2.7, p. 67.

7. M. W. Zemansky, Heat and Thermodynamics, McGraw-Hill, New York, 1943, Table 13.8, p. 245.

8. CRC Handbook of Chemistry and Physics, 36th ed., Chemical Rubber Co., Cleveland, Ohio, 1954, p. 2087.

9. K. Mori, M. Sasakawa, T. Igarashi, Y. Isikawa, K. Sato, K. Noto, and Y. Muto, Physica C 162-164, 512-3 (1989).

10. Cited by E. W. Collings, Second International Symposium on Superconductivity, Tsukuba, Japan, November 14-17, 1989.

11. WADD-TR-60-56, 1960.

12. L. Dresner, Adv. Cryog. Eng. 26, 647-53 (1980).

13. S. Ochiai, K. Hayashi, and K. Osamura, "Influence of Thermal Cycling on Critical Current of Superconducting Silver-Sheathed High $T_{c}$ Wires," Cryogenics 31, 954-64 (1991). 


\section{SYMBOLS}

a Constant defined in Eq. (41)

$B \quad$ Magnetic field

$b \quad$ Constant defined in Eq. (46)

c Dimensionless propagation velocity defined in Eq. (20c)

$C \quad$ Constant defined in Eq. (49); specific heat in Eq. (16a)

$C_{\mathrm{v}} \quad$ Specific heat at constant volume

$E_{1}, E_{2} \quad$ Young's modulus of the silver and the superconductor, respectively

$f, f_{1} \quad$ Volume fraction of silver in the composite

$f_{2} \quad$ Volume fraction of superconductor in the composite

$g \quad$ Function defined in Eqs. (16) and (22)

$h \quad$ Function defined in Eq. (42)

$i \quad$ Operating current divided by critical current

$J \quad$ Overall current density in the conductor

$J_{\mathrm{c}} \quad$ Critical current density of the superconductor

$k \quad$ Thermal conductivity

$k_{*} \quad$ Coefficient defined in Eq. (36)

$L_{0} \quad$ The Lorenz constant, $2.45 \times 10^{-8} \mathrm{~V}^{2} \cdot \mathrm{K}^{-2}$

$Q \quad$ Quench energy

$R \quad$ Universal gas constant, $8.317 \mathrm{~J} / \mathrm{mol} \cdot \mathrm{K}$

$S \quad$ Heat capacity per unit volume

$S_{\mathrm{b}} \quad$ Value of $S$ at $T=T_{\mathrm{b}}$

s $\quad k(d T / d \xi)$; see Eq. (19)

$T \quad$ Temperature

$T_{0} \quad$ Solution for $T$ given in Eq. (41)

$T_{\mathrm{b}} \quad$ Ambient temperature

$T_{\text {cs }} \quad$ Current sharing threshold, defined in Eq. (16)

$T_{\mathrm{hs}} \quad$ Hot-spot temperature

$t \quad$ Time

$V \quad$ Normal zone voltage

$x \quad T / \theta$; later $T / T_{\mathrm{b}}$

$x_{\mathrm{c}} \quad$ Dimensionless critical temperature, $T_{\mathrm{c}} / T_{\mathrm{b}}$

$x_{\mathrm{cs}} \quad$ Dimensionless current sharing threshold, $T_{\mathrm{cs}} / T_{\mathrm{b}}$

$x^{\prime} \quad c^{1 / 3} x$

$y \quad$ Quantity defined in Eq. (9); later, quantity defined in Eq. (20b); later;

function defined in Eq. (41)

$y^{\prime} \quad c^{1 / 3} y$

$z \quad \theta / T$

$\alpha_{1}, \alpha_{2} \quad$ Linear coefficient of thermal expansion of the silver and the superconductor, respectively

$\beta \quad$ Debye coefficient [see Eq. (6a)]

$\gamma \quad$ Sommerfeld constant [see Eq. (6a)]

$\eta_{1}, \eta_{2}$ Strain in the silver and the superconductor, respectively

$\theta \quad$ Debye temperature; temperature rise in Eqs. (32)-(34) 
$\checkmark \quad$ Propagation velocity

$\xi \quad z+v t$

$\rho \quad$ Resistivity

$\rho_{\mathrm{p}} \quad$ Phonon resistivity

$\rho_{\mathrm{r}} \quad$ Residual resistivity

$\rho_{*} \quad$ Coefficient defined in Eq. (36)

$\sigma_{1}, \sigma_{2} \quad$ Stress in the silver and the superconductor, respectively

$\sigma_{y} \quad$ Yield stress of the silver 

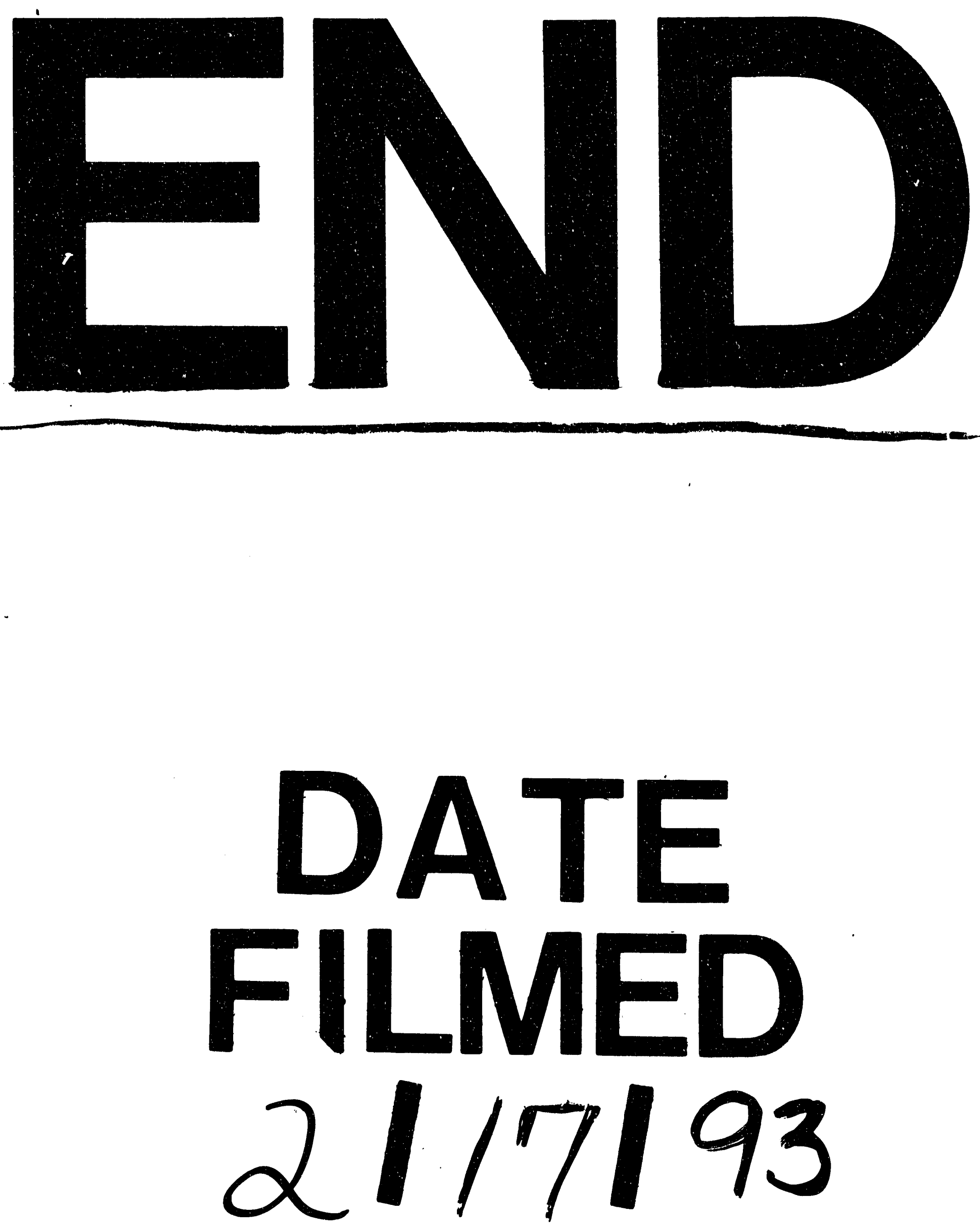
